\title{
II. Die Armee im Staat der SED
}

\section{Die Rolle des Militärs im Marxismus-Leninismus}

$\mathrm{Zu}$ Beginn der marxistischen Theoriebildung entwickelten Karl Marx und Friedrich Engels keine eigene Militärkonzeption ${ }^{1}$. Im „Manifest der Kommunistischen Partei“ von 1848 gingen sie noch davon aus, „daß mit Einführung der sozialistischen Gesellschaftsordnung der Staat sich von selbst auflöst und verschwindet“, wie Engels es später formulierte ${ }^{2}$. Mit dem Verschwinden der Klassengegensätze verliere die „politische Gewalt“ als „organisierte Gewalt einer Klasse zur Unterdrückung einer anderen" ihren Sinn ${ }^{3}$. Implizit setzte dies auch das Verschwinden des Militärs voraus.

Durch die klare Niederlage der Revolutionäre 1848/1849 gelangten Marx und Engels jedoch zu der Erkenntnis, daß die Bewaffnung der Massen allein der Revolution nicht zum Sieg verhelfen würde. Daher integrierten sie Krieg und Militär unter dem Eindruck fehlender Machtmittel zur Durchsetzung ihrer politischen Zielsetzungen dezidiert in die kommunistische Ideologie. Diese wurden zu Instrumenten der revolutionären Strategie und zu Faktoren im gesellschaftlichen Entwicklungsprozeß.

Engels entwickelte in den folgenden zwei Jahrzehnten ein starkes Interesse an militärischen Fragestellungen ${ }^{4}$. Grundsätzlich bewegten sich seine Überlegungen bis 1871 jedoch im Rahmen der zeitgenössischen Armeekonzeptionen. Engels glaubte zwar, daß die „Emanzipation des Proletariats“ in der Zukunft einmal „eine aparte, neue Kriegsmethode erzeugen" werde 5 .Zunächst aber werde die Revolution

1 Vgl. u.a. Höhn, Sozialismus und Heer, S. $31 \mathrm{ff}$., $121 \mathrm{ff}$., $211 \mathrm{ff}$., $339 \mathrm{ff}$.; Jungermann, Die Wehrideologie der SED, S. $29 \mathrm{ff}$. Raymond L. Garthoff hat in diesem Zusammenhang geäußert, der Marxismus habe „die militärische Lehre der Partei oder die des künftigen proletarischen Staates vor 1917 nicht festgelegt oder definiert“. Garthoff, Die Sowjetarmee, S. 49. Jungermann ist dagegen der Auffassung, daß „mit dem Aufbau der Roten Armee faktisch die von Engels 1851 formulierte wehrpolitische Konzeption in die Tat umgesetzt worden" sei. Jungermann, Die Wehrideologie der SED, S. 32. Tatsächlich dürften beide Positionen einseitig überzeichnet sein. Es scheint sowohl eine Kontinuität der Konzeptionen als auch signifikante Kursänderungen als Reaktion auf die aktuelle politische Lage gegeben zu haben. Dies verweist auf das eigentlich zentrale Charakteristikum der marxistisch-leninistischen Militärkonzeption: die Notwendigkeit, den ideologischen Gesellschaftsentwurf mit dem politisch Opportunen zu verbinden.

2 Engels an August Bebel in Zwickau [1875], in: Marx/Engels, Ausgewählte Schriften, Bd. 2, S. $32 \mathrm{f}$.

3 Marx/Engels, Manifest der Kommunistischen Partei, in: Dies., Ausgewählte Schriften, Bd. 1, S. 45.

4 Vgl. Wallach, Die Kriegslehre von Friedrich Engels, S. $17 \mathrm{ff}$

5 Engels, Betrachtungen über die Folgen eines Krieges der Heiligen Allianz gegen Frankreich 
„Krieg zu führen haben mit den Mitteln und nach den Methoden der allgemeinen modernen Kriegführung"6. Um die Anschlußfähigkeit der kommunistischen Ideologie an die gesellschaftliche Wirklichkeit nicht zu gefährden, erteilte er den seiner Ansicht nach unrealistischen „Phantasien von einem Milizheer mit sozusagen gar keiner Dienstzeit" eine Absage7. Eine „aus Zivilisten neuformierte Armee“ sei einem stehenden Heer vor allem „durch die Organisation, die die Hauptstärke der Regulären" bilde8, unterlegen. Erst eine „kommunistisch eingerichtete und erzogene Gesellschaft" könne eine - wenn auch nur „asymptotische“ - Annäherung an das Milizsystem verwirklichen'. Engels verschaffte sich taktisch Entlastung für eine pragmatische Zwischenlösung in der Gegenwart, indem er die Realisierung des Milizgedankens als wenig konkretes Fernziel definierte. Auf diese Weise versuchte er, den erkennbaren Gegensatz zwischen theoretischem Anspruch und praktischer Notwendigkeit zu entschärfen. Die revolutionäre Ideologie und der militärische Fortschritt sollten einander nicht ausschließen.

Engels sprach sich demzufolge für das stehende Heer als Gewaltpotential des Staates nach einer siegreichen Revolution aus. Zweckmäßig sei nur eine „regelmäßige Armee gegen die Invasion" von außen ${ }^{10}$. Zugleich befürwortete er aber auch die allgemeine Wehrpflicht. Er äußerte: „Je mehr Arbeiter in den Waffen geübt werden, desto besser." 11 Im Verlauf des revolutionären Prozesses schlage dann "das Fürstenheer um in ein Volksheer“, was zur „Sprengung ... aller stehenden Armeen von innen heraus" führe ${ }^{12}$.

Dieser herkömmliche Ansatz wurde aber um ein spezifisch neues Element erweitert: die „proletarische Garde ... zum Dienst im Innern"13, die „mit selbstgewählten Chefs und eigenem selbstgewählten Generalstabe" parallel zum Heer existieren sollte ${ }^{14}$. Die Garde stellte aber keinen Entwurf für eine proletarische Armee dar. Vielmehr sollte die Revolution über ein innenpolitisches Machtinstrument zur „Drohung gegen die legitimistischen Gegenden"15 verfügen. Von nun an gehörte der Einsatz der bewaffneten Macht auch gegen Teile der eigenen Bevölkerung zur marxistischen Strategie.

Anders als Engels hatte sich Marx vor 1871 zu militärischen Fragen nur wenig geäußert. Bei ihm jedoch hatte das Milizsystem nicht an Bedeutung verloren. Dessen

im Falle einer siegreichen Revolution im Jahre 1852, in: Ders., Ausgewählte militärische Schriften, Bd. 1, S. 218.

6 Ebenda, S. 221.

7 Engels, Die preußische Militärfrage und die deutsche Arbeiterpartei [1865], in: Ders., Ausgewählte militärische Schriften, Bd. 2, S. 319.

8 Engels, Lehren des amerikanischen Krieges [1861], in: Marx/Engels, Werke, Bd. 15, S. 404.

9 Engels an Marx vom 16. Januar 1868, in: Marx/Engels, Werke, Bd. 32, S. 21.

10 Engels, Betrachtungen über die Folgen eines Krieges, in: Ders., Ausgewählte militärische Schriften, Bd. 1, S. 226.

11 Engels, Die preußische Militärfrage und die deutsche Arbeiterpartei, in: Ders., Ausgewählte militärische Schriften, Bd. 2, S. 331.

12 Engels, Herrn Eugen Dührings Umwälzung der Wissenschaft [1878], S. 177.

13 Engels, Betrachtungen über die Folgen eines Krieges, in: Ders., Ausgewählte militärische Schriften, Bd. 1, S. 226.

14 Marx/Engels, Ansprache der Zentralbehörde an den Bund vom März 1850, in: Dies., Werke, Bd. 7, S. 250.

15 Engels, Betrachtungen über die Folgen eines Krieges, in: Ders., Ausgewählte militärische Schriften, Bd. 1, S. 227. 
Errichtung blieb weiterhin das Ziel einer „allgemeinen Volksbewaffnung“. Die Existenz von „kleinen stehenden Heeren“ begriff Marx nur als Übergangslösung16, um die "allgemeine Ausbildung im Waffengebrauch“ der proletarischen Massen zu ermöglichen ${ }^{17}$.

Der Aufstand der Pariser Kommune 1871 veranlaßte Marx dann aber im Gegensatz zu Engels dazu, einer vornehmlich ideologisch geprägten Militärkonzeption den Vorzug zu geben. In der Pariser Kommune sah Marx seinen revolutionären Gesellschaftsentwurf erstmals zum Teil verwirklicht. Für ihn war damit zugleich auch eine spezifisch eigene Form einer Militärorganisation der Arbeiterklasse geschaffen worden. Die Erfahrungen der Pariser Kommune hätten gezeigt, daß „die Arbeiterklasse ... nicht die fertige Staatsmaschine einfach in Besitz nehmen und diese für ihre eigenen Zwecke in Bewegung setzen“ könne ${ }^{18}$. Vielmehr gelte es, die „bürokratisch-militärische Maschinerie“ zu zerbrechen ${ }^{19}$. Marx befürwortete daher die „Unterdrückung des stehenden Heeres und seine Ersetzung durch das bewaffnete Volk“. Das Gewaltpotential der siegreichen Revolution sollte eine „hauptsächlich aus Arbeitern bestehende Nationalgarde" bilden ${ }^{20}$.

Diesen Ansatz übernahm zunächst auch Wladimir I. Lenin ${ }^{21} 1903$ äußerte er: „Das stehende Heer ist ein Heer, das vom Volke getrennt ist und dafür ausgebildet wird, auf das Volk zu schießen.... Zur Verteidigung des Staates gegen einen feindlichen Überfall braucht man kein stehendes Heer, dazu genügt eine Volkswehr." 22

Die russische Revolution von 1905 veranlaßte Lenin jedoch dazu, seine militärtheoretische Konzeption in rascher Folge zu variieren, um programmatisch nicht den Anschluß an die revolutionäre Entwicklung zu verlieren. Der Aufstand auf dem Panzerkreuzer Potjomkin führte bei ihm zunächst zur Aufgabe des Volksbewaffnungsgedankens. Lenin zielte nun situationsbedingt darauf $a b$, aus Teilen der regulären zaristischen Armee den Kern einer „revolutionären Armee“ zu bilden, die „als Stütze einer revolutionären Regierung dienen" sollte. Er konstatierte, die "großen geschichtlichen Fragen“ könnten „nur durch Gewalt gelöst werden“. Die „Organisation der Gewalt im modernen Kampf aber" sei „eine militärische Organisation"23. Die Armee wurde damit auch bei Lenin ein zentrales Instrument im revolutionären Klassenkampf.

Vor dem Hintergrund der gescheiterten Revolution vollzog Lenin jedoch einen erneuten Positionswechsel. Statt einer Ausnutzung der bestehenden militärischen Strukturen plädierte er wieder für die „Abschaffung des stehenden Heeres und

16 Marx, Instruktionen für die Delegierten des Provisorischen Zentralrats zu den einzelnen Fragen [1867], in: Marx/Engels, Werke, Bd. 16, S. 199.

17 Ebenda.

18 Marx, Der Bürgerkrieg in Frankreich. Adresse des Generalrats der Internationalen Arbeiterassoziation [1871], in: Marx/Engels, Werke, Bd. 17, S. 336.

19 Marx an Ludwig Kugelmann in Hannover (Auszug) [1871], in: Marx/Engels, Ausgewählte Schriften, Bd. 2, S. 433.

20 Marx, Der Bürgerkrieg in Frankreich, in: Marx/Engels, Werke, Bd. 17, S. 338.

21 Vgl. Jacobs, The Leninist Revival in Soviet Military Doctrine, S. $23 \mathrm{ff}$.

22 Lenin, „An die Dorfarmut. Die Ziele der Sozialdemokraten, dargelegt für die Bauern“ [1903], in: Ders., Über Krieg, Armee und Militärwissenschaft, Bd. 1, S. 50.

23 Lenin, Revolutionäre Armee und revolutionäre Regierung [1905], in: Ders., Über Krieg, Armee und Militärwissenschaft, Bd. 1, S. 164. 
seine Ersetzung durch die allgemeine Volksbewaffnung" in Form einer "Volksmiliz"24.

Nach Beginn des Ersten Weltkrieges hielt Lenin zunächst an diesem ideologisch geprägten, auf Entdifferenzierung abzielenden Ansatz fest: Für den Kampf des Proletariats gegen die Bourgeoisie solle eine "proletarische Miliz" geschaffen werden, deren Offiziere durch die Mannschaften zu wählen seien ${ }^{25}$. Mit Bezug auf Marx forderte Lenin, die alte „Staatsmaschine“ durch eine neue zu ersetzen, in der „Polizei, Armee und Bürokratie mit dem bis auf den letzten Mann bewaffneten Volk zu einer Einheit verschmolzen“ seien $^{26}$. Eine solche „wirkliche Volksmiliz" werde das „ausführende Organ der ,Sowjets der Arbeiter- und Soldatendeputierten"“27. Lenins Militärkonzeption blieb bis zur Oktoberrevolution 1917 deutlich innenpolitisch geprägt. Wichtigstes Ziel der „Bewaffnung des Proletariats“ war weiter, die „Bourgeoisie zu besiegen, zu expropriieren und zu entwaffnen" 28 .

Auch jetzt glaubte Lenin noch daran, daß die Revolution den Sieg erringen werde, wenn sie sich in ihrer Militärorganisation konsequent an der Pariser Kommune als „Keimform der Sowjetmacht“ orientiere ${ }^{29}$. Die oberste Aufgabe blieb für ihn weiterhin die „Abschaffung des stehenden Heeres“30. Die neue „sozialistische Rote Armee“, so Lenin, werde die angestrebte „allgemeine Volksbewaffnung“ verwirklichen, indem „neue Kader der Roten Garde“ die „werktätigen Massen für den bewaffneten Kampf" schulten ${ }^{31}$. Die Behauptung, stehende Heere seien aufgrund der „Komplizierung des öffentlichen Lebens“ oder der „Differenzierung der Funktionen" alternativlos, bezeichnete er als Phrase ${ }^{32}$. Nach der Entmachtung der Bourgeoisie beginne der Staat abzusterben. Folglich konstatierte Lenin: „An Stelle besonderer Institutionen einer bevorzugten Minderheit (... des stehenden Heeres) kann das die Mehrheit selbst unmittelbar besorgen ... . "33 Im „harten Bürgerkrieg“" werde dann "die neue Militärorganisation der neuen Klasse" geschaffen ${ }^{34}$.

Parallel dazu verfestigte sich jedoch Lenins Einsicht in die militärischen Defizite der im Aufbau befindlichen Roten Armee. Er mußte einräumen, daß die „Offensive des imperialistischen Deutschlands gegen die Sozialistische Sowjetrepublik“ am Ende des Ersten Weltkrieges „eine bittere, kränkende, harte, aber notwendige, nütz-

24 Ebenda.

25 Lenin, Das Militärprogramm der proletarischen Revolution [1916], in: Ders., Werke, Bd. 23, S. $80 \mathrm{f}$.

26 Lenin, Briefe aus der Ferne. Brief 3. Über die proletarische Miliz [1917], in: Ders., Werke, Bd. 23, S. 340.

27 Ebenda, S. $342 \mathrm{f}$.

28 Lenin, Das Militärprogramm der proletarischen Revolution, in: Ders., Werke, Bd. 23, S. 76.

29 Lenin, Bericht über die Tätigkeit des Rats der Volkskommissare, 11. (24.) Januar [1918], in: Ders., Werke, Bd. 26, S. 455.

30 Lenin, Beratung der Regimentsvertreter der Petrograder Garnison. Rede über die Herstellung der Ordnung in der Stadt [1917], in: Ders., Werke, Bd. 26, S. 264.

31 Lenin, Bericht über die Tätigkeit des Rats der Volkskommissare, in: Ders., Werke, Bd. 26, S. $462 \mathrm{f}$.

32 Lenin, Staat und Revolution. Die Lehre des Marxismus vom Staat und die Aufgaben des Proletariats in der Revolution [1918], in: Ders., Werke, Bd. 25, S. 401.

33 Ebenda, S. 432.

34 Lenin, Die proletarische Revolution und der Renegat Kautsky [1918], in: Ders., Werke, Bd. 28, S. 284. 
liche, wohltätige Lehre" gewesen sei ${ }^{35}$. Hier deutete sich Lenins zunehmende Hinwendung zu einer pragmatischeren Militärkonzeption an.

Es waren schließlich aber wohl vor allem die Erfahrungen des Bürgerkrieges in Rußland nach Ende des Ersten Weltkrieges, die eine Zäsur in Lenins militärtheoretischer Auffassung bewirkten. Der existentiellen Bedrohung der bolschewistischen Herrschaft durch die „weißen“ Truppen konnte nur mit einer verbesserten militärischen Leistungsfähigkeit der Roten Armee begegnet werden. Dafür aber mußte Lenin einige seiner bisherigen Leitsätze korrigieren. Jetzt forderte er gezwungenermaBen die „Ausnutzung jenes Vorrats an bürgerlicher Wissenschaft und Technik des Militarismus“ durch die proletarische Klasse, „ohne die sie die moderne Technik und die modernen Methoden der Kriegführung nicht meistern" könne ${ }^{36}$. Lenin schränkte aber ein, daß es „ein nicht wiedergutzumachender Fehler" wäre, „deshalb die Frage der Änderung der Grundlagen unserer Militärpolitik zu stellen“37.

Bei der Begründung seines neuen Konzeptes konstruierte Lenin eine Prämisse, um seinen Positionswechsel abzusichern. Er äußerte: „Die Frage der Struktur der Roten Armee war eine völlig neue Frage, sie war nicht einmal theoretisch gestellt worden. "38 Damit umging Lenin die Gefahr, sich in einen Widerspruch zu der bestehenden Lehrmeinung zu begeben.

Strukturfragen ergaben sich für Lenin dabei zunächst vor allem hinsichtlich des Einsatzes von "bürgerlichen Spezialisten" in der Roten Armee ${ }^{39}$. Auch hier behauptete er, die „früheren Lehrer des Sozialismus“ hätten sich zu dieser Frage nicht geäußert: „Sie existierte nicht für sie, denn sie entstand erst, als wir uns an den Aufbau der Roten Armee machten." 40 Diese Argumentation war ebenso taktisch wie dialektisch. Denn Engels etwa hatte sich für das stehende Heer vor allem deshalb ausgesprochen, weil er glaubte, die Revolution würde ohne bürgerliches Fachwissen nicht bestehen können. Nur dadurch, daß Lenin diese bestehenden Überlegungen konsequent ignorierte, erhielt er die Möglichkeit, eine sachlich zwingende Maßnahme als revolutionäre Neuerung in die Ideologie zu integrieren.

Noch Ende 1918 hatte Lenin gefordert, „beim Aufbau der neuen Armee die Kommandeure nur aus dem Volk" zu rekrutieren ${ }^{41}$. Doch schon auf dem VIII. Parteitag der Kommunistischen Partei im März 1919 vertrat er das neue Modell: Den Rumpf des Offizierskorps der Armee der Arbeiterklasse sollten nun in großer Zahl ehemals zaristische Offiziere bilden. Lenin verteidigte diese Kehrtwendung: In der „Frage der Militärfachleute“ bekämpfe die Partei die „scheinbar radikale, in Wirklichkeit aber von Unwissenheit zeugende Einbildung ..., als wären die Werktätigen imstande, den Kapitalismus und die bürgerliche Ordnung zu überwinden, ohne von den bürgerlichen Spezialisten zu lernen, ohne sie auszunutzen"42. Ohne Militär-

35 Lenin, Eine harte, aber notwendige Lehre [1918], in: Ders., Werke, Bd. 27 , S. 47.

36 Lenin, VIII. Parteitag der KPR(B), 18.-23. März 1919. Bericht des Zentralkomitees, 18. März, in: Ders., Werke, Bd. 29, S. 139.

37 Lenin, Alle zum Kampf gegen Denikin! (Brief des ZK der KPR [Bolschewiki] an die Parteiorganisationen) [1919], in: Ders., Werke, Bd. 29, S. 438.

38 Lenin, VIII. Parteitag der KPR(B), in: Ders., Werke, Bd. 29, S. 137.

39 Ebenda, S. 141.

40 Ebenda, S. 139.

41 Lenin, Rede am „Tag des roten Offiziers“, 24. November 1918, in: Ders., Werke, Bd. 28, S. 190.

42 Lenin, Alle zum Kampf gegen Denikin!, in: Ders., Werke, Bd. 29, S. 439. 
fachleute hätte "jene Rote Armee“ nicht "geschaffen werden können, die ... glänzende Siege ... zu erringen" vermocht habe ${ }^{43}$.

Mit der Integration der zaristischen Offiziere in die Rote Armee erhielt zwangsläufig die Frage der politischen Kontrolle besondere Bedeutung. Wichtige Kontrollinstrumente waren für Lenin die „verstärkte politische Arbeit in der Armee“ und die „Arbeit der Kommissare“, die die „Konzentrierung der allseitigen Kontrolle über den Kommandeurstab ... in den Händen der Arbeiterklasse" garantieren sollten ${ }^{44}$. Die Leitung der Armee sollte letztlich ausschließlich durch die „Avantgarde, die führende Partei des Proletariats" erfolgen ${ }^{45}$.

Entscheidenden Einfluß auf die Weiterentwicklung der kommunistischen Militärkonzeption hatte nach der Errichtung des bolschewistischen Regimes Leo Trotzki, der im März 1918 Kriegskommissar geworden war. Im Zuge des Aufbaus der Roten Armee schuf er die grundlegenden Rahmenbedingungen für die zivil-militärischen Beziehungen in sozialistischen Staaten ${ }^{46}$. Trotzki sah sich dabei gezwungen, trotz massiver innerparteilicher Kritik das militärisch Nötige mit den ideologischen Vorgaben zu verbinden.

Er war sich dieser „Zwiespältigkeit" durchaus bewußt, die er aber mit der „Übergangsperiode von der Herrschaft der Bourgeoisie zur sozialistischen Gesellschaftsordnung" rechtfertigte ${ }^{47}$. In seinen Thesen zur Frage der Armeebildung äußerte Trotzki auf dem VIII. Parteitag 1919, daß der Kampf gegen den „inneren und äußeren Klassenfeind“ es verhindert habe, auf „,organischem“ Wege zu einer Arbeiterund Bauernmiliz zu kommen". Wenn es die innen- und außenpolitische Lage jedoch zulasse, solle zu einem territorial gegliederten „Klassenmilizheer" übergegangen werden ${ }^{48}$. Nach Etablierung der sozialistischen Ordnung in allen Ländern werde man schließlich ein "communist people in arms" werden ${ }^{49}$.

Nach Ansicht von Trotzki war die Schaffung einer proletarischen „Klassenarmee" wegen der militärischen Zwangslage zunächst aber unausweichlich gewesen. Diese begriff er jedoch nur als einen „Übergangstypus“: Dem „sozialen Bestande“ nach sei sie ein „Klassenheer", den Ausbildungsmethoden entsprechend ein „,stehendes', ,reguläres" Heer"50. Letzteres bedeutete aber ausdrücklich nicht, daß das Militär „außerhalb der Politik“ stehen sollte ${ }^{51}$. Trotzki stellte vielmehr klar, der

43 Ebenda, S. 438.

44 Ebenda, S. 440 .

45 Lenin, IX. Parteitag der KPR (B), 29. März-5. April 1920. Bericht des Zentralkomitees, 29. März, in: Ders., Werke, Bd. 30, S. 440.

46 Vgl. Deutscher, Trotzki, S. $382 \mathrm{ff} ., 446 \mathrm{ff}$.

47 Trotzki, Die Rote Armee. Rede, gehalten in der Sitzung des Allrussischen Zentralexekutivkomitees am 22. April 1918, in: Ders., Die Geburt der Roten Armee, S. 43.

48 Trotzki, Unsere Politik in der Frage der Armeebildung. Thesen, angenommen auf dem VIII. Parteitag der KPR, im März 1919, in: Ders., Die Geburt der Roten Armee, S. 168 f.

49 Trotsky, Theses on going over to the militia system (For the Ninth Congress of the Russian Communist Party [1920], in: How the Revolution Armed, vol. 2, S. 192.

50 Trotzki, Unsere Politik in der Frage der Armeebildung, in: Ders., Die Geburt der Roten Armee, S. 166, 168.

51 Trotzki, Die Schaffung der Roten Arbeiter- und Bauernarmee. Bericht auf dem 5. SowjetKongreß in der Sitzung vom 10. Juli 1918, in: Ders., Die Geburt der Roten Armee, S. 86. 
„revolutionäre Charakter der Armee" werde letztlich „durch den Charakter des Sowjetregimes bestimmt" 52 .

Durch diesen Kunstgriff wurde es Trotzki möglich, spezifisch militärische Fragen zu entideologisieren. In diesem Sinne stand es für ihn außer Zweifel, daß die Rote Armee als eine „army of the professional type“ zu organisieren war, wollte Sowjetrußland in der Lage sein, einen drohenden Angriff imperialistischer Staaten abzuwehren ${ }^{53}$. In Anlehnung an Engels äußerte er: „Wir brauchen nicht eine Armee, die auf irgendeinem selbst ausgetüftelten Prinzip aufgebaut ist, sondern ... eine ... zentralisierte Armee, die auf den Prinzipien der Kriegswissenschaft und Technik basiert." 54 Auf Trotzkis Betreiben wurde so das Leitbild professioneller Streitkräfte dauerhaft in die marxistisch-leninistische Militärkonzeption integriert.

Durch die Eingliederung ehemals zaristischer Offiziere als Folge des Mangels an militärisch qualifizierten Bolschewisten entstand für Trotzki die Notwendigkeit, einen die militärische Truppenführung kontrollierenden Parteiapparat in der Armee zu installieren. Während die Militärfachleute allein in „rein militärischen, operativen Fragen" Entscheidungen treffen sollten ${ }^{55}$, war es Aufgabe der Kommissare, „alle militärischen Befehle“ zu sanktionieren ${ }^{56}$. Dieser zweigeteilte Kommandoapparat war für Trotzki als „Organisationstypus nicht ideal“57, nichtsdestoweniger wurde er durch den Beschluß des VIII. Parteitages im März 1919 in der Roten Armee etabliert. Neben den Kommissaren waren die „kommunistischen Zellen“ und die „politischen Abteilungen" die zentralen Strukturelemente des Parteiapparates ${ }^{58}$. Der Parteiapparat erwies sich jedoch nur als vermeintliche Übergangslösung. Obwohl er immer wieder partiell modifiziert wurde, blieb er in seiner grundsätzlichen Ausformung bis zum Ende der UdSSR bestehen 59 .

Trotzkis zweigleisige, auf Parteikontrolle und Professionalität basierende Militärkonzeption wurde zwischen 1921 und 1924 von Teilen der Partei und der Armee massiv attackiert. Seine Gegner, deren herausragender Protagonist General Michail W. Frunse war, vertraten die Existenz einer eigenständigen, spezifisch proletari-

52 Trotzki, Unsere Politik in der Frage der Armeebildung, in: Ders., Die Geburt der Roten Armee, S. 171.

53 Trotsky, Report to the Conference of military-education institutions of the Moscow Military District, December 12, 1921, in: How the Revolution Armed, vol. 4, S. 139.

54 Trotzki, Die Schaffung der Roten Arbeiter- und Bauernarmee, in: Ders., Die Geburt der Roten Armee, S. 96.

55 Trotzki, Die Rote Armee, in: Ders., Die Geburt der Roten Armee, S. 30.

56 Trotzki, Unsere Aufgabe [1918], in: Ders., Die Geburt der Roten Armee, S. 25.

57 Trotzki, Die Rote Armee, in: Ders., Die Geburt der Roten Armee, S. 30.

58 Trotzki, Unsere Politik in der Frage der Armeebildung, in: Ders., Die Geburt der Roten Armee, S. 170.

59 Graduelle Veränderungen gab es besonders im Zusammenhang mit der Funktion der Politstellvertreter der Einheiten. Diese ersetzten nach 1924 sukzessive die politischen Kommissare, nachdem das ZK in einem prinzipiellen Beschluß die ungeteilte Befehlsbefugnis in den Streitkräften eingeführt hatte. Bis dahin hatten die Kommissare die Befehle aller Kommandeure gegenzeichnen müssen. Die Politstellvertreter übten dagegen nur eine indirekte Kontrolle gegenüber der militärischen Truppenführung aus. Die Kommissare ersetzten dann nochmals 1937-1940 sowie um 1941/1942 die Politstellvertreter; ihre Funktion wurde im Anschluß jedoch nicht zuletzt wegen der Ineffizienz der Doppelspitze im Zweiten Weltkrieg wieder suspendiert. Vgl. Colton, Commissars, Commanders, and Civilian Authority, S. 14, $44 \mathrm{f}, 87 \mathrm{ff}$. 
schen Militärkonzeption und Kriegslehre ${ }^{60}$. Trotzki verurteilte diese „denigration of bourgeois science or bourgeois strategy “61. Es sei ein Fehler zu glauben, „a revolutionary Army, based upon consciousness, upon political, revolutionary élan, had no need of regulations"62. Die Vorstellung, die Revolution habe eine proletarische, der bürgerlichen überlegene Militärkonzeption hervorgebracht, war für Trotzki nicht mehr als eine "uncritical idealisation“ der bolschewistischen Kriegsführung im Bürgerkrieg. Er lehnte daher die Entwicklung einer „so-called, unified military doctrine “" als Ergebnis der "generalisation of a new revolutionary strategy and tactics" entschieden $a^{63}$. Diese barg für Trotzki die latente Gefahr einer Radikalisierung und Entdifferenzierung der Streitkräfte, wodurch jedoch die Einsatzfähigkeit der Roten Armee als professionelle Militärorganisation wieder in Frage gestellt worden wäre.

Frunse seinerseits hatte auf dem X. Parteikongreß der Kommunistischen Partei im März 1921 eigene Thesen für eine „einheitliche Militärdoktrin“ aufgestellt ${ }^{64}$. Diese Konzeption sah die Umwandlung der Roten Armee in einen „einheitlichen Organismus“ vor. Dieser, so Frunse, solle „von oben bis unten nicht nur durch die Gemeinsamkeit der politischen Ideologie“ gekennzeichnet sein, „sondern auch durch die Einheitlichkeit der Anschauungen über den Charakter der ... militärischen Aufgaben, der Methoden ihrer Lösung und der Methoden der militärischen Ausbildung der Truppen" 65 .

Grundsätzlich akzeptierte Frunse zwar die Verwendung derselben „Formen und Methoden, deren sich auch die bürgerlichen Armeen bedienten"66. Er forderte aber, daß ihre Adaption von vornherein ,auf der allgemeinen Grundlage der marxistischen Lehre" erfolgen solle ${ }^{67}$. Dementsprechend begriff er die spezifische Organisation der Roten Armee, so etwa ihre „politischen Abteilungen ..., deren Rolle als einer Waffe besonderer Art, ... die innere Struktur der Armee, die neuen Ideen über Disziplin, das Verhältnis zwischen den Kommandeuren und den Soldaten usw." nicht als pragmatisches Instrument zur Machtsicherung, sondern bereits als genuinen Teil einer proletarischen Militärkonzeption ${ }^{68}$.

Der Konflikt um die einheitliche Militärdoktrin erlebte seinen Höhepunkt auf dem XI. Parteikongreß im März 1922. Frunse gelang auch hier keine überzeugende

$60 \mathrm{Vgl}$. Jacobs, Frunze, S. $19 \mathrm{ff}$.

61 Trotsky, Report. To the Eleventh Congress of the Russian Communist Party (Bolsheviks), March 29, 1922, in: How the Revolution Armed, vol. 4, S. 186.

62 Trotsky, Speech at a meeting of cadres of the 1st Unified Military School named after the AllRussia Central Executive Committee, November 2, 1921, in: How the Revolution Armed, vol. 4, S. 95.

63 Trotsky, Report to the Eleventh Congress of the Russian Communist Party, in: How the Revolution Armed, vol. 4, S. $186 \mathrm{ff}$.

64 Frunse, Die Reorganisierung der Roten Armee [1921], in: Ders., Ausgewählte Schriften, Bd. 1, S. 138.

65 Ebenda.

66 Frunse, Die augenblicklichen militärischen Hauptaufgaben. Aus dem Referat und aus dem Schlußwort auf der Beratung der Armeedelegierten des XI. Parteitages der KPR(B) [1922], in: Ders., Ausgewählte Schriften, Bd. 1, S. 213.

67 Frunse, Die Reorganisierung der Roten Armee, in: Ders., Ausgewählte Schriften, Bd.1, S. 138.

68 Frunse, Die augenblicklichen militärischen Hauptaufgaben, in: Ders., Ausgewählte Schriften, Bd. 1, S. 213. 
Konkretisierung seiner Konzeption. Als Frunse Trotzki 1925 als Volkskommissar für Militär- und Marineangelegenheiten sowie als Vorsitzender des Revolutionären Kriegsrates ablöste, endete die Debatte. Nach der Entmachtung Trotzkis entfiel die machtpolitische Rivalität in Militärkreisen als ein Motiv der Konfrontation ${ }^{69}$. Im Anschluß kam es zu keiner Revision der von Trotzki maßgeblich entwickelten sowjetischen Militärkonzeption. Vielmehr blieb die Parallelstruktur von regulärer Armee und Parteiapparat auch weiterhin bestehen ${ }^{70}$.

\section{NVA und Offizierskorps in der Militärkonzeption der SED 1956-1965}

Die militärtheoretischen Grundlagen des Marxismus-Leninismus und die Rahmenbedingungen der zivil-militärischen Beziehungen in der UdSSR konditionierten grundsätzlich auch die Militärkonzeption der SED und das prinzipielle Verhältnis zwischen der Partei und dem Militär in der DDR im Jahrzehnt nach der Gründung der NVA ${ }^{71}$. Wie die KPdSU, so vertrat auch die SED die Doktrin, daß das Militär im Verlauf der Transformation der Gesellschaft hin zum Kommunismus überflüssig werde. Die Existenz sozialistischer Streitkräfte, so Heinz Hoffmann, ergebe sich "nicht aus den Gesetzmäßigkeiten der sozialistischen Produktionsweise"72. Eine Armee sei vielmehr auch im Fall der DDR wegen der "geschichtlichen Erfahrungen“ notwendig, die besagten, daß das „Proletariat nach seiner Machtergreifung gezwungen" sei, die „Errungenschaften der proletarischen Revolution und des sozialistischen Aufbaus im Innern und nach aussen zu verteidigen"73. Hoffmann konstatierte jedoch für die Zukunft: „Die innere Funktion der sozialistischen Streitkräfte verliert im Prozeß des Aufbaus des Sozialismus immer mehr an Bedeutung und erlischt mit der Vollendung des Sozialismus ganz und gar." Dann würden sich die „sozialistischen Streitkräfte“ von einem „Klasseninstrument der siegreichen Arbeiterklasse“ zu einer „Armee des ganzen Volkes zum Schutz des sozialistischen Vaterlandes" wandeln" ${ }^{74}$.

Während der „Entfaltung des sozialistischen Aufbaus“ war die NVA nach Ansicht der SED als Teil der Gesellschaft jedoch „in diesen Umwandlungsprozeß aktiv

69 So hatte Frunse vor der „Schmälerung der Verdienste der kommunistischen Elemente innerhalb der Armee" gewarnt. Frunse, Die augenblicklichen militärischen Hauptaufgaben, in: Ders., Ausgewählte Schriften, Bd. 1, S. 221.

70 Sowohl die in den zwanziger und dreißiger Jahren neben der regulären Armee existierenden Milizelemente als auch die 1939 eingeführte allgemeine Wehrpflicht resultierten aus einer finanziellen beziehungsweise sicherheitspolitischen Zwangslage, bedeuteten aber keine grundlegende Änderung der Konzeption. Vgl. Gosztony, Die Rote Armee, S. $107 \mathrm{ff} ., 168 \mathrm{ff}$.

71 Vgl. u.a. Blanke, Zum Verhältnis Militär - Partei - Gesellschaft in der DDR, S. $190 \mathrm{ff}$.; Blanke, NVA und Innere Sicherheit, S. $134 \mathrm{ff}$.

72 Hoffmann, Grundfragen der Militärpolitik der Sozialistischen Einheitspartei Deutschlands. Vorlesung an der Parteihochschule „Karl Marx" beim Zentralkomitee der SED, 4. März 1964, in: Ders., Sozialistische Landesverteidigung, T. 1, S. 127.

73 Entwurf des Referats des Ministers für Nationale Verteidigung für die Eggersdorfer Tagung, 1957, SAPMO-BArch, DY 30/IV 2/12/10, Bl. 1.

74 Hoffmann, Grundfragen der Militärpolitik, in: Ders., Sozialistische Landesverteidigung, T. 1, S. 128. 
eingeschlossen"75. Hoffmann wies dem Militär dabei vor allem eine pädagogische Aufgabe zu: „Im sozialistischen Staat hat der Dienst in den Streitkräften große Bedeutung für die sozialistische Erziehung der Jugend und damit für die innere Festigung der sozialistischen Gesellschaft." 76

Die Theorie vom provisorischen Charakter des Militärs modifizierte die SED 1967 jedoch im Kern, als eine baldige Entwicklung der Gesellschaft hin zum Kommunismus offensichtlich unwahrscheinlich geworden war. Das militärische Gewaltpotential wurde fortan als "organischer Bestandteil“ des sozialistischen Gesellschaftssystems aufgefaßt ${ }^{77}$. Mit der Festigung des sozialistischen Staates werde auch das Militär immer stärker werden ${ }^{78}$.

Mittelfristig wurde die Existenz des Militärs zunächst jedoch vor allem durch die äußere Bedrohung der DDR und den internationalen Klassenkampf legitimiert. Nach Ansicht der SED belegten dies die historischen Erfahrungen der internationalen Arbeiterbewegung und der sozialistischen Staaten. Hoffmann stellte dementsprechend fest, daß die „äußere Funktion“ des Militärs und damit „die Existenz der sozialistischen Armeen überhaupt" notwendig sei, „solange es noch eine mächtige Gruppe imperialistischer Staaten" gebe ${ }^{79}$. Das Politbüromitglied Hermann Matern bemerkte dazu, die kapitalistischen Staaten zielten darauf ab, „die alten Verhältnisse ... wiederherzustellen“. Aus diesem Grund sei ,jedes sozialistische Land verpflichtet ..., seinen militärischen Schutz zu organisieren und sich entsprechende bewaffnete Kräfte zu schaffen" 80 .

Äußerungen des ersten Ministers für Nationale Verteidigung der DDR Willi Stoph lassen darüber hinaus die in den fünfziger Jahren zumindest intern erwogene radikale Instrumentalisierung der Armee im Rahmen der politischen Konzeption der SED erkennen. Diese widersprach der Politik der „friedlichen Koexistenz“, die in dieser Phase bereits die sowjetische Außenpolitik bestimmte ${ }^{81}$. Neben den Verteidigungsaufgaben nach außen und dem „Kampf gegen konterrevolutionäre Kräfte

75 Entwurf der Thesen über die neue Entwicklungsetappe der NVA und die sich daraus ergebenden Aufgaben, 1961, SAPMO-BArch, DY 30/IV 2/12/15, Bl. 147.

76 Hoffmann, Grundfragen der Militärpolitik, in: Ders., Sozialistische Landesverteidigung, T. 1, S. 128.

77 Hoffmann, Der VII. Parteitag, S. 757.

78 Hoffmann begründete diesen programmatischen Wechsel damit, daß zum „Wesensinhalt des entwickelten gesellschaftlichen Systems des Sozialismus" neben der Wirtschaft auch eine „starke sozialistische Staatsmacht" gehöre, die die Gesellschaftsordnung schützen könne. Beide Komponenten bildeten „eine untrennbare Einheit“, ein „geschlossenes Gesamtsystem“, das die "vollendete sozialistische Gesellschaftsordnung" darstelle. Die Landesverteidigung und die Entwicklung der Armee seien daher „untrennbar mit der gesamten gesellschaftlichen Entwicklung ... verbunden“. Hoffmann erklärte: „Die Einordnung unserer militärischen Funktion in das gesellschaftliche Gesamtsystem des Sozialismus bedeutet eine neue Stufe der Einheit von Politik, Ökonomie und Landesverteidigung. "Hoffmann, Die gesellschaftliche Entwicklung in der Deutschen Demokratischen Republik bis zur Vollendung des Sozialismus und die wachsende Verantwortung der Nationalen Volksarmee für den zuverlässigen Schutz unseres souveränen sozialistischen Vaterlandes. Referat auf der Aktivtagung der Nationalen Volksarmee zur Auswertung des VII. Parteitages der SED, 2. Mai 1967, in: Ders., Sozialistische Landesverteidigung, T. 2, S. $534 \mathrm{f}$.

79 Hoffmann, Grundfragen der Militärpolitik, in: Ders., Sozialistische Landesverteidigung, T. 1, S. 128.

80 Matern, Die Führung der Nationalen Volksarmee durch die SED, S. 198.

81 Vgl. Heller/Nekrich, Geschichte der Sowjetunion, Bd. 2, 1940-1980, S. 251 f. 
im Innern des Landes" 82 wies Stoph der NVA eine weitere Funktion zu. Er sagte: „Die Nationale Volksarmee ist ein Machtinstrument der in unserer Republik unter Führung der Partei herrschenden Arbeiter-und-Bauern-Klasse. Ihr Charakter und ihre Aufgaben werden deshalb von den politischen Zielen des Kampfes der Arbeiterklasse bestimmt.“ Diese seien darauf gerichtet, „die Macht des Militarismus und Imperialismus in ganz Deutschland zu beseitigen und in ganz Deutschland den Sozialismus aufzubauen“. Das sei aber „nur im langwierigen[,] harten Klassenkampf ... zu erreichen“, wobei die DDR, „in der bereits der Sozialismus aufgebaut" werde, „die staatliche Basis dieses Kampfes" sei. Die NVA „als Instrument des Klassenkampfes" müsse ihre „Aufgaben im Interesse des Kampfes der Arbeiterklasse ... erfüllen" ${ }^{83}$. Hier erhielt die NVA eindeutig eine über die Verteidigungsaufgaben hinausgehende, offensive Rolle zugewiesen. Diese Funktion des Militärs dürfte die SED-Führung bis zum Mauerbau durchaus als opportun erachtet haben. Das Motiv für diese Position war vermutlich die massive Bedrohungsperzeption, die die Politik des Regimes während dieser Zeit umfassend dominierte.

In Übereinstimmung mit der Militärkonzeption der UdSSR war es für die SED folgerichtig, die NVA als ,reguläre, den Anforderungen eines modernen Krieges entsprechende Armee" zu konzipieren ${ }^{84}$. Demzufolge sollten die DDR-Streitkräfte „nach den allgemeinen Grundsätzen des modernen Militärwesens ... aufgebaut und organisiert" werden ${ }^{85}$. Bei der Gründung der NVA orientierte man sich am Leitbild einer professionellen und spezialisierten Militärorganisation. Dieser Ansatz war flexibel genug, um entsprechend der jeweiligen politischen Lage nach 1956 in Verbindung mit dem Freiwilligenprinzip und nach 1961 mit der Wehrpflicht realisiert zu werden.

Anscheinend existierte während der Phase verstärkter politischer Durchdringung der NVA in den fünfziger Jahren durchaus auch die Auffassung in der SED, den Primat der Ideologie auf alle Bereiche der Militärkonzeption auszudehnen. So ließ Matern 1958 eine Distanzierung von der in Teilen entideologisierten Militärkonzeption Trotzkis erkennen, als er konstatierte, die Partei habe „feste wissenschaftliche Auffassungen zu allen militärischen Fragen, die mit den wissenschaftlichen Lehren des Marxismus-Leninismus“ übereinstimmten. Demzufolge seien „die wissenschaftlichen Erkenntnisse der Armee von der Wissenschaft des Marxismus-Leninismus abgeleitet und ihr untergeordnet“. Daher lägen „allen militärischen Fragen ... die Erkenntnisse der Partei zugrunde" $" 86$. Im Rahmen der Verhinderung der von der SED vermuteten latenten Autonomisierungstendenzen innerhalb der NVA erfolgte zeitweilig anscheinend eine zumindest verbale Orientierung an der „einheitlichen Militärdoktrin" Frunses.

Die SED tendierte in der Aufbauphase der NVA offenbar teilweise dazu, in ihrer Militärkonzeption eigene Ansätze zu formulieren. Die theoretischen und prakti-

82 Entwurf des Referats des Ministers für Nationale Verteidigung für die Eggersdorfer Tagung, 1957, SAPMO-BArch, DY 30/2/12/10, Bl. 2.

83 Protokoll der Beratung leitender Parteifunktionäre in der NVA mit Mitgliedern des Politbüros des ZK der SED in Eggersdorf, 12./13. Juni 1957 [zit.: Protokoll der Eggersdorfer Tagung, 12./13. Juni 1957], BA-MA, VA-P-01/037, Bl. $43 \mathrm{f}$.

84 Protokoll der Eggersdorfer Tagung, 12./13. Juni 1957, BA-MA, VA-P-01/037, Bl. 7.

85 Ebenda, Bl. 44.

86 Matern, Die Führung der Nationalen Volksarmee durch die SED, S. 201. 
schen Vorgaben der UdSSR wurden daher ausdrücklich „schöpferisch“ angewandt. So postulierte die Partei, daß es „zweifelsohne“ bei der Organisierung des militärischen Schutzes „Besonderheiten“ gebe, die „aus den konkreten Bedingungen der Lage und der Entwicklung der DDR“ resultierten. Diese Besonderheiten, die „naturgemäß auch Auswirkungen auf den Aufbau, die Struktur, die Organisation und die Ausbildung und Erziehung in der Armee" hätten, gelte es „gründlich zu erforschen und in der täglichen Arbeit zu berücksichtigen" 87 . Eine solche Position stellte unverhohlen das militärtheoretische Interpretationsmonopol der KPdSU in Frage. Sie ist zudem ein deutlicher Hinweis auf die auch innerhalb des sozialistischen Gesellschaftssystems bestehenden Differenzierungstendenzen.

Für die SED war die NVA eine Armee „neuen Typus“88 - die „erste wahrhafte Volksarmee in der Geschichte Deutschlands" 89 . Nach Auffassung der Partei bedeutete dies eine Zäsur in der historischen Dimension. In der NVA sollte es zum ersten $\mathrm{Mal}$ „keinen militärischen Kommißgeist“ mehr geben ${ }^{90}$. Nach Ansicht der SED existierte nunmehr kein Gegensatz mehr zwischen Bevölkerung und Armee. Stoph stellte dementsprechend fest: „Die Angehörigen der Nationalen Volksarmee sind Blut vom Blute unseres werktätigen Volkes. "91 Die NVA sollte folglich geprägt sein durch den „Patriotismus" der Armeeangehörigen, ihre feste „Verbundenheit mit den Werktätigen ..., besonders mit der Arbeiterklasse, zum sozialistischen Internationalismus, zur Freundschaft mit allen Völkern“ sowie „zur unverbrüchlichen Freundschaft mit der Sowjetunion und den Armeen der volksdemokratischen Staaten“. Die SED verlangte zudem den „unversöhnlichen Haß“ der Armeeangehörigen "gegen die westdeutschen Militaristen, gegen den Imperialismus" und alle „Feinde“ des Volkes" ${ }^{92}$ sowie ihre „unerschütterliche Treue zur Partei" ${ }^{\text {"93. }}$

Konstitutiv für die zivil-militärischen Beziehungen in der DDR war der Führungsanspruch der SED in der NVA. Die Partei leitete diesen theoretisch von ihrer historischen Rolle als „Vorhut der Arbeiterklasse“ im Gesamtprozeß der gesellschaftlichen Umgestaltung ab. Die „Diktatur des Proletariats“ werde „durch die marxistisch-leninistischen Parteien verwirklicht“, die die „führende Rolle beim sozialistischen Aufbau“ verkörperten ${ }^{94}$. Stoph bemerkte, daß es keine „höhere Organisation des Proletariats als die Partei“ gebe, weshalb sie auch die „Führung in der Volksarmee verwirklicht" habe. Er folgerte daraus: „Eine Trennung in militärische und politische Führung ist deshalb nicht zulässig, weil die militärische Tätigkeit im Interesse der Politik des proletarischen Staates erfolgt und ihr untergeordnet ist. "95

Der theoretische Führungsanspruch der SED konditionierte demzufolge auch die Stellung der Streitkräfte innerhalb der Gesellschaft. Matern äußerte: „Im Arbeiter-und-Bauern-Staat nimmt die Armee eine besonders wichtige Stellung ein. Aber

87 Thesen der KPdSU - ein wichtiges Lehrbuch, S. 4.

88 Bogisch, Über die militärpolitische Bedeutung der 30. Tagung des ZK, S. 156.

89 Protokoll der Eggersdorfer Tagung, 12./13. Juni 1957, BA-MA, VA-P-01/037, Bl. 8.

90 Stenographische Niederschrift der 3. Parteikonferenz der SED, 24.-30. März 1956, SAPMO-BArch, DY 30/IV 1/3/6, Bl. 834.

91 Ebenda.

92 Bestimmungen für die Arbeit der Politorgane, 1958, S. $31 \mathrm{f}$.

93 Instruktion für die Arbeit der Parteiorganisationen, 1958, S. 6.

94 Matern, Die Führung der Nationalen Volksarmee durch die SED, S. 199.

95 Protokoll der Eggersdorfer Tagung, 12./13. Juni 1957, BA-MA, VA-P-01/037, Bl. 45. 
sie hat niemals das Recht, sich über oder neben die Partei und den Staat zu stellen." Jegliche „Bestrebungen, die führende Rolle der Partei zu negieren“, müßten die weitere Entwicklung „unweigerlich" schädigen ${ }^{96}$. Politische Neutralität und institutionelle Autonomie der NVA hätten sowohl den ideologischen Grundprinzipien als auch den politischen Zielen und der konkreten Machtsicherung der SED widersprochen.

Aus diesem Grund maß die SED der „Entwicklung eines spezifisch sozialistischen Offizierskorps " 97 beim Aufbau der NVA besondere Bedeutung bei ${ }^{98}$. Dabei propagierte die Partei im Rahmen ihrer Militärkonzeption den „Typ des sozialistischen Offiziers"99, der einen radikalen Bruch mit der Militärtradition darstellen sollte. Nach Ansicht der SED gab es erstmals in einer deutschen Armee keine Klassengegensätze mehr zwischen Offizieren und Soldaten. Die Offiziere seien „Söhne der Arbeiterklasse", der sie zugleich dienten ${ }^{100}$. Sie bildeten mit den Soldaten eine "politisch-moralische und militärische Einheit", die durch das kameradschaftliche Verhältnis der „Klassenbrüder"101 und nicht durch Befehl und Gehorsam geprägt sei. Hoffmann äußerte, deshalb könne es „keine Trennung zwischen Offizieren und Soldaten geben". Vielmehr müßten sie "gemeinsam arbeiten, lernen, leben“ sowie „sich gegenseitig achten und helfen"102. Nach Auffassung der SED unterschieden sich die NVA-Angehörigen "lediglich nach Aufgaben und Verantwortung, die ihnen entsprechend ihrer Befähigung, ihrer Erfahrung und Eignung von Partei und Regierung übertragen wurden"103.

Der Offizier "neuen Typs" 104 sollte sich zudem durch vorbildliches Verhalten und seine „Verbindung zum werktätigen Volk"105 auszeichnen. Für die SED war das Leitbild des Offiziers vor allem politisch motiviert: Dieser habe die „Pflicht und Ehre, die ihm unterstellten Soldaten im Sinne des Marxismus-Leninismus zu erziehen und ... so auszubilden, dass sie in jeder Situation treu zu ihrem sozialistischen Vaterland" stünden ${ }^{106}$. Das Ziel der SED war es, die Entstehung eines traditionellen und nicht politisch geprägten korporativen Selbstverständnisses des NVA-Offizierskorps zu verhindern. Sie versuchte statt dessen, ein neues, ideologisch begründetes Leitbild zu konstruieren, das seinerseits jedoch nicht ohne den traditionellen

${ }^{96}$ Matern, Die Führung der Nationalen Volksarmee durch die SED, S. 199.

${ }^{97}$ Entwurf des Referats des Ministers für Nationale Verteidigung für die Eggersdorfer Tagung, 1957, SAPMO-BArch, DY 30/IV 2/12/10, B1. 10.

98 Vgl. Ilter, Die sozialistische Offizierspersönlichkeit, S. $1543 \mathrm{ff}$.

${ }^{99}$ Protokoll der 2. Tagung der II. Delegiertenkonferenz der Parteiorganisationen der SED in der NVA, 25./26. März 1959, BA-MA, VA-P-01/556, Bl. 224.

100 Entwurf des Referats des Ministers für Nationale Verteidigung für die Eggersdorfer Tagung, 1957, SAPMO-BArch, DY 30/IV 2/12/10, Bl. 21.

101 Matern, Die Führung der Nationalen Volksarmee durch die SED, S. 200.

102 Protokoll der 2. Tagung der II. Delegiertenkonferenz der Parteiorganisationen der SED in der NVA, 25./26. März 1959, BA-MA, VA-P-01/556, Bl. 67.

103 Die Lehren aus der Entwicklung der deutschen und internationalen Arbeiterbewegung und des Marxismus-Leninismus über die Rolle, Bedeutung und Entwicklung der Kader und ihre Anwendung für die Arbeit mit den Kadern in der NVA, 1959, SAPMO-BArch, DY 30/IV 2/12/26, Bl. 59 .

104 Glaser, Militärpolitik für Sozialismus und Frieden, S. 46.

105 Entwurf des Referats des Ministers für Nationale Verteidigung für die Eggersdorfer Tagung, 1957, SAPMO-BArch, DY 30/IV 2/12/10, Bl. 22.

106 Ebenda, Bl. 21. 
Ehrbegriff auskam, der jedoch neu interpretiert wurde. So äußerte Stoph, es gebe „auch in einer Arbeiter- und Bauernarmee eine Offiziersehre“. Doch sei „Ehre ... nicht gleich Ehre, genauso wie Gewehr nicht gleich Gewehr" sei. Das sei eine „Klassenfrage“, und eine „wirkliche Offiziersehre“ könne es „nur in einer sozialistischen Armee geben"107.

Dies implizierte jedoch nicht, daß die SED den sozialistischen Offizier einseitig als proletarischen Klassenkämpfer begriff. Vielmehr sollte er gemäß der Theorie des Marxismus-Leninismus und der praktischen Erfahrungen der KPdSU zugleich auch den Typ des professionellen Soldaten verkörpern. Um beide Bestandteile zu dem intendierten Idealtyp generieren zu können, postulierte die SED die „Einheit von politischer und militärischer Führung": In der Person des sozialistischen Offiziers sollten sich gleichermaßen "gute militärische Kenntnisse und Fertigkeiten mit sozialistischem Bewusstsein und Klassenverbundenheit" paaren ${ }^{108}$. Stoph stellte in diesem Zusammenhang fest: „Es genügt nicht schlechthin Militärspezialist zu sein. Von jedem ... Offizier ... muss verlangt werden, dass er ein politischer Funktionär und Erzieher ist, der die Entwicklungsgesetze der Gesellschaft richtig begreift und sich bei seiner praktischen Tätigkeit davon leiten läßt." "109 Jeder Offizier sollte in der Lage sein, „seine militärischen Aufgaben mit der Lösung der politischen Fragen zu verbinden" 110 . Als militärischer Führungskader und „Diener der Arbeiterklasse“ war der Offizier nach Ansicht der SED daher sowohl an die Beschlüsse der Partei als auch an die der Regierung gebunden, die er in der Armee zu verwirklichen hatte ${ }^{111}$. Rudolf Dölling, der 1958-1959 Stellvertreter des Ministers für Nationale Verteidigung und Chef der Politischen Verwaltung war, sagte: „Diese Forderung wird an jeden Offizier gestellt, ganz gleich, ob er Mitglied der Partei ist oder nicht. Deshalb muß jeder Offizier ein überzeugter Sozialist werden oder sein ... " 112

Obwohl die SED das „Klassenprinzip als oberstes Prinzip bei der Aufstellung des Offizierskorps" postulierte113, legitimierte die Praxis der Bolschewiki beim Aufbau der Roten Armee die Eingliederung ehemaliger Wehrmachtsoffiziere als „klassenfremde Militärspezialisten“" auch in die bewaffneten Verbände der DDR nach 1948, um fehlenden militärischen Sachverstand zu kompensieren ${ }^{114}$. Dies wurde aber nicht zuletzt aufgrund ihrer vergleichsweise kleinen Zahl nach der NVA-Gründung 1956 in der Militärkonzeption der SED prinzipiell weder öffentlich noch theoretisch begründet.

107 Ebenda, Bl. $20 \mathrm{f}$.

108 Ebenda, Bl. 7.

109 Rede des Genossen Minister bei der Eröffnung der Schulung der leitenden Generale und Offiziere, 17. Februar 1958, BA-MA, VA-01/5043, Bl. 109.

110 Hinweise zur Auswertung der 2. Tagung der Delegiertenkonferenz der NVA. Protokoll Nr. 9/59 der Leitungssitzung der Politischen Verwaltung des Ministeriums für Nationale Verteidigung, 4. April 1959, BA-MA, VA-P-01/023, Bl. 44.

111 Engels, Die führende Rolle der Partei verstärken, S. 285.

112 Protokoll der Delegiertenkonferenz der Parteiorganisationen der SED in der NVA, 24.-26. Juni 1958, BA-MA, VA-P-01/038, Bl. 32.

113 Teilbeitrag der Verwaltung Kader zur Einarbeitung in die Gesamtanalyse und Grundkonzeption der Entwicklung der NVA, 10. März 1967, BA-MA, VA-01/5679, Bl. 4.

114 Vgl. Wenzke, Auf dem Weg zur Kaderarmee, S. $220 \mathrm{ff}$. 